\begin{tabular}{|c|c|}
\hline Title & A phase diagram of neutral polyampholyte : from solution to tough hydrogel \\
\hline Author(s) & $\begin{array}{l}\text { Ihsan, A bu Bin; Sun, Tao Lin; Kuroda, Shinya; Haque, Md. A namul; Kurokawa, Takay uki; Nakajima, Tasuku; Gong, } \\
\text { Jian Ping }\end{array}$ \\
\hline Citation & $\begin{array}{l}\text { Journal of Materials Chemistry B, 1(36), 4555-4562 } \\
\text { https://doi.org/10.1039/c3tb20790k }\end{array}$ \\
\hline Issue Date & $2013-09$ \\
\hline Doc URL & http:/hdl .handle.net/2115/58074 \\
\hline Rights & embargo12ヶ月あり (2014.9以降) \\
\hline Type & article (author version) \\
\hline File Information & A bu Bin Ihsan-JMC-B manuscript-1.pdf \\
\hline
\end{tabular}

Instructions for use 


\section{A Phase Diagram of Neutral Polyampholyte - From Solution to Tough Hydrogel}

Abu Bin Ihsan ${ }^{1}$, Tao Lin $\mathrm{Sun}^{2}$, Shinya Kuroda ${ }^{2}$, Md. Anamul Haque ${ }^{3}$,Takayuki

$$
\text { Kurokawa }^{3} \text {, Tasuku Nakajima }{ }^{3} \text {, Jian Ping Gong* }{ }^{3}
$$

${ }^{1}$ Transdisciplinary Life Science Course, Graduate School of Life Science, Hokkaido University, Sapporo 060-0810, Japan

${ }^{2}$ Department of Biological Sciences, Graduate School of Science, Hokkaido University, Sapporo 060-0810, Japan

${ }^{3}$ Faculty of Advanced Life Science, Hokkaido University, Sapporo 060-0810, Japan *Authors to whom correspondence should be addressed.

Tel\&FAX: +81-(0)11-706-2774, E-mail: gong@ mail.sci.hokudai.ac.jp. 


\section{Abstract}

Our recent study has revealed that neutral polyampholytes form tough physical hydrogels above a critical concentration $C_{\mathrm{m}, \mathrm{c}}$ by forming ionic bonds of wide strength distribution. In this work, we systematically investigate the behavior of a polyampholyte system, poly(NaSS-co-DMAEA-Q), randomly copolymerized from oppositely charged monomers, sodium $p$-styrenesulfonate (NaSS) and acryloyloxethyltrimethylammonium chloride (DMAEA-Q) without and with a slight chemical cross-linking. A phase diagram of formulation has been constructed in the space of monomer concentration $C_{\mathrm{m}}$ and cross-linker density $C_{\mathrm{MBAA}}$. Three phases are observed for the as-synthesized samples: homogeneous solution at dilute $C_{\mathrm{m}}$, phase separation at semi-dilute $C_{\mathrm{m}}$, and homogenous gel at concentrated $C_{\mathrm{m}}$. Above a critical $C_{\mathrm{m}, \mathrm{c}}$, the polyampholyte forms supramolecular hydrogel with high toughness by dialysis of the mobile counter-ions, which substantially stabilizes both the intraand inter chain ionic bonds. Presence of chemical cross-linker $\left(C_{\mathrm{MBAA}}>0\right)$ brings about a shift of the tough gel phase to lower $C_{\mathrm{m}}$. . The tough polyampholyte gel, containing $\sim 50 \mathrm{wt} \%$ water, is highly stretchable and tough, exhibits fracture stress of $\sigma_{\mathrm{b}} \sim 0.4 \mathrm{MPa}$, fracture strain of $\varepsilon_{\mathrm{b}} \sim 30$, and the work of extension at fracture $\sim 4 \mathrm{MJ} / \mathrm{m}^{3}$. These values are in the level of most tough soft materials. 
Owing to the reversible ion bonds, the poly(NaSS-co-DMAEA-Q) gels also exhibit complete self-recovery (100\%) and high fatigue resistance upon repeated large deformation.

Key Words: Polyampholyte, Hydrogel, Sacrificial bond, Ion bond, Toughness,

Self-recovery 


\section{Introduction}

Hydrogels are a class of promising soft and wet materials for their wide applications as cell scaffolds in tissue engineering, smart actuators, and sensors in industry. ${ }^{1-8}$ However, the application of the hydrogels had been largely limited by their mechanical weakness. The inventions of several strong and tough hydrogels in the early 2000s have greatly expanded the potential possibility of this material ${ }^{9-13}$ and initiated many researches to develop high strength hydrogels. ${ }^{14-21}$ These studies have also stimulated the fundamental researches on the fracture mechanics of soft and wet materials. ${ }^{22-23}$ Studies on the double-network (DN) gels, one of the toughest hydrogels consisting of interpenetrating brittle and ductile networks, have indicated that the internal rupture of the brittle network, which effectively dissipates energy and prevents catastrophic crack propagation upon loading, gives the extraordinarily high toughness. ${ }^{24-28}$ The studies on the DN hydrogel revealed a novel principle to toughen the materials, that is, an easily fractured, brittle internal structure makes the material as a whole mechanically tough. ${ }^{24-28}$ Thus, the brittle network acts as a 'sacrificial bond', similar to the proposed model for toughening of bones. ${ }^{29-30}$ This sacrificial bond principle, accordingly, suggests a new strategy for designing high-strength materials: incorporating, on purpose, a mechanically fragile structure to toughen the material as a whole.

As the internal rupture of a DN gel is due to the irreversible breaks of the covalent 
bonds of the brittle network, a DN gel shows Mullins effect and degenerates gradually after repeated deformation. To address this problem, several recent works have replaced the covalent bonds with non-covalent bonds to allow the sacrificial bond to be regenerated. ${ }^{15,20,31}$ Though studies along these lines have produced much tougher hydrogels as compared to conventional ones, most of these systems only showed partial recovery after internal rupture. ${ }^{15,31}$

Polyampholytes are polyelectrolytes carrying opposite charges randomly distributed. Neutral polyampholyte of equal amount of opposite charges, due to intra-chain ion bonds formation, forms globule structure and precipitates in dilute aqueous solution of low ionic strength. ${ }^{32-33}$ Chemically cross-linked polyampholyte hydrogels deswell near the equal charge composition. ${ }^{34-37}$ Recently, we developed a novel class of physical hydrogels from linear polyampholytes by employing the ion bond as a reversible sacrificial bond that breaks and reforms dynamically. ${ }^{38}$ Owing to the random distribution, the opposite charges on the polyampholytes form multiple ion bonds of wide distribution in strength. The strong bonds work as permanent cross-linker, imparting the elasticity of the hydrogel. The weak bonds are fragile and they break under stress, serving as reversible sacrificial bonds. Upon rupture of the weak bonds, the globule polymer chain unfolded, serving as hidden length. These two processes 
dissipate energy and impart toughness to the hydrogel, in analogy to that of bone toughening in which $\mathrm{Ca}^{2+}$ ion rupture and protein unfolding dissipate energy. ${ }^{22-23}$

The polyampholyte gels, belonging to supramolecular hydrogels and containing 50wt\% water, are very tough and viscoelastic. For example, the P(NaSS-co-MPTC) physical hydrogels, randomly copolymerized from concentrated aqueous solutions of oppositely charged monomers, sodium $p$-styrenesulfonate (NaSS) and 3-(methacryloylamino)propyl-trimethylammonium chloride (MPTC), exhibit high strength (tensile fracture stress $\sigma_{\mathrm{b}}=1.8 \mathrm{MPa}$ ), high extensibility (tensile fracture strain $\varepsilon_{\mathrm{b}}=7.4$ ), and high toughness (tearing energies $T=4000 \mathrm{~J} / \mathrm{m}^{2}$ ). These values are ranked among the highest level of soft materials, either synthetic such as tough DN hydrogels, filled rubbers, or natural, such as cartilages. ${ }^{22,39,40}$ In contrast to the DN hydrogels, in which covalent bonds of the brittle network break and could not recover, the polyampholyte hydrogels are self-recoverable due to the non-covalent ion bonds. In addition to these excellent mechanical properties, these polyampholyte hydrogels have excellent biocompatibility and anti-biofouling properties. ${ }^{38}$ As the materials have a wide spectrum of excellent mechanical properties even in physiological solutions; they have high potential as structural biomaterials. Also, the anti-biofouling property suggests the potential use in hygiene and medical fields. This ion bond approach has great advantages 
for practical applications: it is general and directly applicable to diverse ionic combinations, and the one-step polymerization process is simple and easy to scale-up in industry. The polyampholyte approach opens a new avenue to synthesize tough soft and wet materials.

In the previous Communication, we have shortly reported that the mechanical properties of the polyampholyte hydrogels are strongly dependent on the combinations of the ionic monomer pairs. More hydrophilic monomers produced softer and stretchable gels and vice versa. ${ }^{38}$ In this paper, we focus on the polyampholyte P(NaSS-co-DMAEA-Q) system synthesized from sodium $p$-styrenesulfonate (NaSS) and acryloyloxethyltrimethylammonium chloride (DMAEA-Q)(Scheme 1). The latter is relatively hydrophilic in comparison with the cationic monomer MPTC used in our previous report. $^{38}$ 


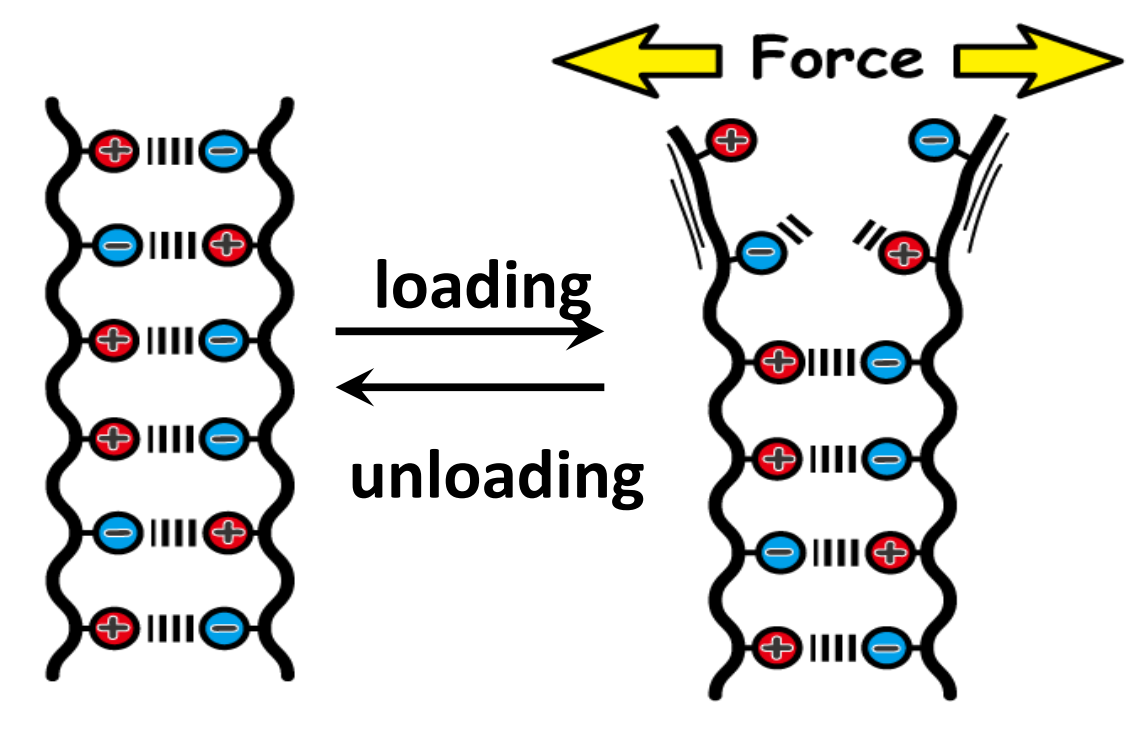



NaSS (-)<smiles>C=CC(=O)OCC[N+](C)(C)Cl</smiles>

DMAEA-Q (+)

Scheme 1. Schematic illustration of the dynamic and reversible ionic bonds (weak bonds) formed by the random copolymerization of oppositely charged monomers at the charge balance point. Below: the chemical structure of anionic monomer NaSS and cationic monomer DMAEA-Q to form polyampholyte gels P(NaSS-co-DMAEA-Q).

Neutral polyampholytes form globule structure in aqueous medium by formation of ionic bonds, which leads to phase separation. In this work, we first determine the optimized charge ratio of the precursor solution to form the neutral polyampholyte. Then we investigate the effects of the monomer concentration $C_{\mathrm{m}}$ and the chemical cross-linker density $C_{\mathrm{MBAA}}$ on the behavior of the polyampholyte. These two parameters 
are crucial to control the phase separation of the system. A phase diagram of formulation in the $C_{\mathrm{m}}-C_{\mathrm{MBAA}}$ space is constructed and homogenous hydrogel phase is determined. Furthermore, the tensile behaviors of gels equilibrated in pure water are investigated to determine the tough hydrogel phase. Finally, the hysteresis and self-recovery of the toughest sample is investigated.

\section{Experimental}

\section{Materials}

Anionic monomer, sodium $p$-styrenesulfonate (NaSS; Wako Pure Chemical Industries, Ltd.), cationic monomer, acryloyloxyethyltrimethylammonium chloride (Aron DA-C/DMAEA-Q; 79.0wt\%, MT AquaPolymer,Inc.), chemical cross-linker, $N$, N'-methylene-bis-acrylamide (MBAA; Wako Pure Chemical Industries, Ltd.), and UV initiator, $\alpha$-ketoglutaric acid (Wako Pure Chemical Industries, Ltd.), were used as received. $\mathrm{NaCl}$ (Wako Pure Chemical Industries, Ltd.) used to control the ion strength in the monomer solution, was also used as received. Millipore de-ionized water was used in all of the experiments.

\section{Sample synthesis}

Series 1 .For the experiment to optimize the charge ratio of the polyampholyte gels, the sample was synthesized from precursor aqueous solution containing $2.0 \mathrm{M}$ monomer $C_{\mathrm{m}}$ 
$\left(C_{\mathrm{m}}=[\mathrm{NaSS}]+[\mathrm{DMAEA}-\mathrm{Q}]\right)$ with molar fractions of the anionic monomer $f$ $\left(f=[\mathrm{NaSS}] / C_{\mathrm{m}}\right)$ in the range of $0.44-0.56,0.1 \mathrm{~mol} \%$ chemical cross-linker density $C_{\text {MBAA }}$, $0.1 \mathrm{~mol} \%$ initiator $\alpha$-ketoglutaric acid (both in relative to $C_{\mathrm{m}}$ ), and $0.5 \mathrm{M} \mathrm{NaCl}$.

Series 2.For the experiment to construct the phase diagram in $C_{\mathrm{m}}-C_{\mathrm{MBAA}}$ space, the polyampholyte was synthesized from precursor aqueous solution containing prescribed amount of monomer concentration $C_{\mathrm{m}}\left(C_{\mathrm{m}}=[\mathrm{NaSS}]+[\mathrm{DMAEA}-\mathrm{Q}]\right)$ at the charge balance point $\left(f=0.52\right.$ in feed), $0-0.3$ mol\% chemical cross-linker density $C_{\mathrm{MBAA}}, 0.1 \mathrm{~mol} \%$ initiator $\alpha$-ketoglutaric acid (both in relative to $C_{\mathrm{m}}$ ), and $0.5 \mathrm{M} \mathrm{NaCl}$. The precursor solution was inject into a reaction cell $(10 \mathrm{~cm} \times 10 \mathrm{~cm})$ consisting of a pair of parallel glass plates separated by silicone spacer with various thickness $(\sim 1-3 \mathrm{~mm})$. The precursor solution was then purged in argon atmosphere to remove dissolved oxygen and polymerization was carried out in argon atmosphere in which the oxygen concentration was less than $0.1 \mathrm{ppm}$ by irradiating UV light (UVP lampXX-15BLB, wavelength $365 \mathrm{~nm}$, light intensity $4 \mathrm{~mW} / \mathrm{cm}^{2}$ ) from both side of the reaction cell for $8 \mathrm{hrs}$. The samples were coded with the formulation values of $f, C_{\mathrm{m}}$, and $C_{\mathrm{MBAA}}$ as $f-C_{\mathrm{m}}-C_{\mathrm{MBAA}}$ after the names of the copolymers.

\section{Dialysis and swelling measurement of gels}

The samples in gel phase were taken out from the reaction cell and were immersed in 
de-ionized water at room temperature for at least one week, and the water was changed every day. During this process, mobile counter ions were dialyzed, and the gels reached equilibrium swelling in water. Weights of swollen and dried samples were measured by moisture balance (Moisture balance; MOC-120H, SHIMADZU Co.). Before weighing the weight of swollen gel, the excess water on the gel surface was gently absorbed by using a kimwipe. The drying sample was obtained by evaporate the water in the gel at an elevated temperature of $(30-120)^{\circ} \mathrm{C}$. The weight swelling ratio $\left(Q_{\mathrm{w}}=W / W_{\mathrm{dry}}\right)$, which is the inverse of the polymer weight fraction in the gel, was calculated from the weight ratio of the swollen gel to the dried gel. The length and width of the gel was measured by using a slide calipers. The thickness of gel sample was measured by a Rheometer (Rheometric Scientific). The volume swelling ratio, $Q_{\mathrm{v}}$, is defined as $Q_{\mathrm{v}}=\left(t / t_{0}\right)^{3}$, where $t$ and $t_{0}$ are the thickness of the samples after immersing in pure water and at the as-prepared state, respectively.

\section{Elemental analysis}

In order to determine the true charge compositions in the gels, elemental analysis was performed on samples of series 1. After reaching equilibrium swelling in water, the samples were cut into very small pieces, weighed and then dried in the freeze dryer by using liquid nitrogen. Then, the weights of gels after drying were recorded and the dried 
samples were made into powder. The powdered samples were preserved in small containers. Elemental analyses were carried out at the Center for Instrumental Analysis, Hokkaido University. The amount of carbon $(\mathrm{C})$, hydrogen $(\mathrm{H})$, and nitrogen $(\mathrm{N})$ in percent weight of the gel was determined by the differential thermal conductivity method using CHN Corder MT-6 (Yanaco) analyzer. Sulfur (S) was quantified by the flask combustion method using DionexDX-500 ion chromatography. The true molar fractions of NaSS $\left(\mathrm{C}_{8} \mathrm{H}_{7} \mathrm{SO}_{3}\right)$ in the gels $\left(f_{\text {true }}\right)$ were calculated by considering the pair of $\mathrm{C}, \mathrm{S} ; \mathrm{C}, \mathrm{N}$; and $\mathrm{N}, \mathrm{S}$ and took average values.

\section{Mechanical analysis}

After reaching swelling equilibrium, the tensile and compressive tests were performed with a commercial test machine (Tensilon RTC-1310A, Orientec Co.). For tensile test, the gels, 2.8 8 $\mathrm{mm}$ in thickness depending on the composition, were cut into a dumbbell shape standardized as IEC-540 sizes $(4 \mathrm{~mm}$ in inner width and $20 \mathrm{~mm}$ in gauge length) with a gel cutting machine (Dumb Bell Co., Ltd.). For unconfined, uniaxial compressive test, the gels, $2.8 \sim 11.3 \mathrm{~mm}$ in initial height or thickness, were cut into a disc shape standardized as RRK-1051-02 size of $15 \mathrm{~mm}$ diameter, and were sandwiched between the two parallel plates of Tensilon. The friction between the gel and the plates during compression was reduced by coating the plates with silicone oil. 
The deformation velocity was maintained as $100 \mathrm{~mm} / \mathrm{min}$ and $0.5 \mathrm{~mm} / \mathrm{min}$, respectively, for the tensile and compressive test. The elastic modulus, $E$, was calculated from the slope of the stress-strain curves at the small strain (within 10\%). The work of extension for fracture $W_{\text {ext }}\left(\mathrm{J} / \mathrm{m}^{3}\right)$, a parameter characterizing the work required to fracture the sample, was calculated from the area below the tensile stress-strain curves until sample failure.

\section{Hysteresis and cyclic test}

Hysteresis was observed by performing the tensile loading to a predefined strain and immediately unloading to zero strain at a fixed strain increasing/decreasing velocity of $100 \mathrm{~mm} / \mathrm{min}$. Multiple loading and unloading cycles at a fixed strain at various waiting times of 0 to 30 minutes between two successive cycles for the same sample were performed. All the loading-unloading cycles were performed in water to prevent water evaporation from samples.

\section{Results and discussion}

\section{Structure optimization}

In order to form stable ionic association, the charge balance of the polyampholyte gel is the most crucial parameter. ${ }^{34-37}$ A series of polyampholyte gels were prepared by varying the NaSS monomer fraction $f$ around the charge balance point over a range of $0.44-0.56$, 
while keeping the total monomer concentration $C_{\mathrm{m}}$ and the chemical cross-linker density as a constant $\left(C_{\mathrm{m}}=2.0 \mathrm{M}, C_{\mathrm{MBAA}}=0.1 \mathrm{~mol} \%\right)$. After reaching equilibrium swelling in water, the gels with $f$ of $0.51-0.525$ shrank comparing with their as-prepared state $\left(Q_{\mathrm{v}}<1\right)$, whereas the gels with $f$ deviated from this range swelled $\left(Q_{\mathrm{v}}>1\right)$ (Fig. 1a). The shrinking of the gels near the charge balance point indicates the ion bonds (ion complex) formation between the oppositely charged immobile ions in water. By the complex formation, the mobile counter-ions release from the hydrogel to the external water medium (dialysis process). On the other hand, the swelling for the gels with large charge imbalance is dominantly induced by the counter-ion osmosis effect of the net polymer charges, the same as like-charged polyelectrolyte gels.

Although the $Q_{\mathrm{v}}$ showed the similar minimum values in the range of $0.51-0.525$, both the elastic modulus $(E)$ and the compressive fracture stress $\left(\sigma_{\mathrm{com}}\right)$ of the gels showed the maximum at $f=0.52$ (Fig. 1a). This suggests that $f=0.52$ is the true charge balanced point. This assumption was justified by the result of the elemental analysis of the samples. As shown in Fig. 1b, the true NaSS fraction in the gels, $f_{\text {true, }}$ is slightly deviated from their nominal value $f$, and the gel with an exactly balanced charge $\left(f_{\text {true }}=0.5\right)$ is synthesized from the precursor solution of $f=0.52$.

To further investigate the mechanical behaviors of the gel near the charge balance point, 
the tensile test of the samples with $f_{\text {true }}$ around 0.5 were performed. The stress-strain curve of the samples with $f_{\text {true }}$ close to 0.50 exhibited very high tensile fracture stress $(\sim 0.4 \mathrm{MPa})$ and strain $(\sim 3000 \%)\left(\right.$ Fig. 1c). In sharp contrast, the samples with $f_{\text {true }}$ slightly deviated from 0.50 showed poor tensile fracture stress and strain (Fig. 1c). This indicates that a stoichiometric ion complex formation is required to form mechanically strong polyampholyte hydrogels $\mathrm{P}(\mathrm{NaSS}-\mathrm{co}$-DMAEA-Q). The effect of charge imbalance to the mechanical performance of gels is not symmetric. For the same amount of net charge, NaSS-poor samples showed a much better mechanical performance than that of the NaSS-rich samples (Fig. 1c). 



(c)

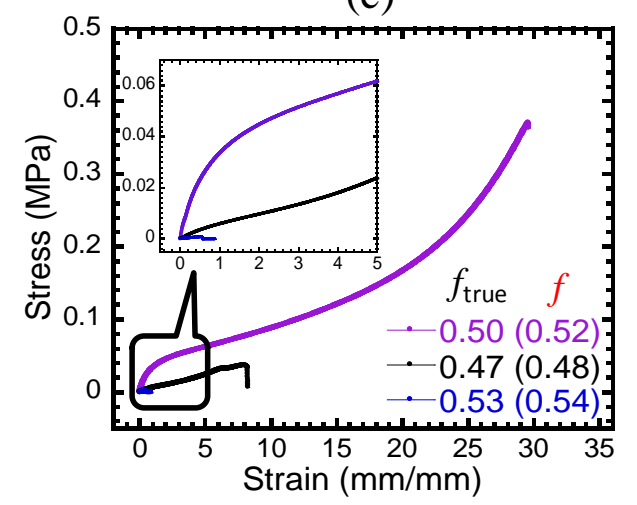

Fig. 1.(a) The effect of negative charge fraction $(f)$ on the volume swelling ratio (blue), $Q_{\mathrm{v}}$, the weight swelling ratio (red), $Q_{\mathrm{w}}$, Young's modulus (black), $E$, and compressive fracture stress (green), $\sigma_{\text {com }}$, of polyampholyte gels synthesized at total monomer concentration $C_{\mathrm{m}}=2.0 \mathrm{M}$ and cross-linker density $C_{\mathrm{MBAA}}=0.1 \mathrm{~mol} \%$. Results above the dashed blue line correspond to the swelling $\left(Q_{\mathrm{v}}>1\right)$, while below that to the shrinking $\left(Q_{\mathrm{v}}<1\right)$ of the gels in water. (b) The true NaSS molar fraction, $f_{\text {true, }}$ as a function of NaSS molar fraction in feed, $f$. The black line corresponds to the ideal case that $f=f_{\text {true. }}$ The true stoichiometric charge ratio $\left(f_{\text {true }}=0.5\right)$ was obtained at $f=0.52$. (c) Tensile stress-strain curves of the polyampholyte hydrogels synthesized at $C_{\mathrm{M}}=2.0 \mathrm{M}, C_{\mathrm{MBAA}}=$ $0.1 \mathrm{~mol} \%$, and various charge fraction $f$. Error bars in (a) and (b) are standard deviations from at least 3 samples. 
As shown in Fig. 1a, the weight swelling ratio $Q_{\mathrm{w}}=W / W_{\text {dry }}$ of the gel at the equilibrium state in water $W$ and the dried state $W_{\mathrm{dry}}$, is above 2even at the charge balanced point of $f_{\text {true }}=0.5$ where the gel shrank to the minimum value. This indicates that the neutral polyampholyte gel still can hydrate and the water content is maintained as high as $\sim 50 w t \%$. This value is quite close to that of biological tissues, ${ }^{41}$ but much lower than that of the conventional hydrogels or DN gels that have a much higher water content $(>85 w t \%)^{42}$

Hereafter, we investigated the effect of other structure parameters on the mechanical properties of the polyampholyte gels at the equi-molar monomer fraction $f=0.52$ $\left(f_{\text {true }}=0.5\right)$. The total monomer concentration $C_{\mathrm{m}}(\mathrm{M})$ substantially influences the behaviors of the samples. Fig. 2a shows images of as-prepared samples that were synthesized at various $C_{\mathrm{m}}$ but constant $f=0.52$ and $C_{\mathrm{MBAA}}=0.1 \mathrm{~mol} \%$. At very low monomer concentrations $\left(C_{\mathrm{m}}<0.3 \mathrm{M}\right)$, the solution kept transparency with no precipitation even after UV-irradiation for a long time. This indicates that short polymer chains were formed at the low monomer concentration, which does not cause precipitation. In the $0.3 \mathrm{M}<C_{\mathrm{m}}<1.0 \mathrm{M}$, precipitation appeared and strong phase separation occurred, indicating the intra-chain ion complex formation. At concentration range of $1.0 \mathrm{M}<C_{\mathrm{m}}<1.3 \mathrm{M}$, uniform gel phase was formed, but the gels swelled in 
water and were mechanically weak. Above a critical concentration $C_{\mathrm{m}, \mathrm{c}}=1.6 \mathrm{M}$, the gels shrank in water and showed very high toughness. Therefore, the stable ion complex only forms at very high polymer concentration, probably stabilized by the entanglement of polymer chains. At $C_{\mathrm{m}}>2.8 \mathrm{M}$, the monomer was not completely soluble due to limited solubility. Addition of chemical cross-linker brings about a shift of gel phase to low $C_{\mathrm{m}, \mathrm{c}}$ values. Fig. $2 \mathrm{~b}$ summarized the behaviors of the samples in the $C_{\mathrm{m}}-C_{\mathrm{MBAA}}$ diagram for $f=0.52$, where $C_{\mathrm{MBAA}}$ is the cross-linker density (mol\%) in relative to $C_{\mathrm{m}}$. Phase separation is a time dependent process and so whether it occurs will depend on the relative rates of polymerization and phase separation. So, strictly speaking, the phase diagram shown in Figure $\mathbf{2 b}$ is a kinetic diagram that depends on the polymerization kinetics. In the present case, as revealed by kinetic study of ${ }^{1} \mathrm{H}-\mathrm{NMR}$, more than $90 \%$ of the monomers converted into polymers within the first 100 minutes (data is not shown), although the UV irradiation was lasted for $8 \mathrm{hrs}$ during the sample synthesis. 
(a)

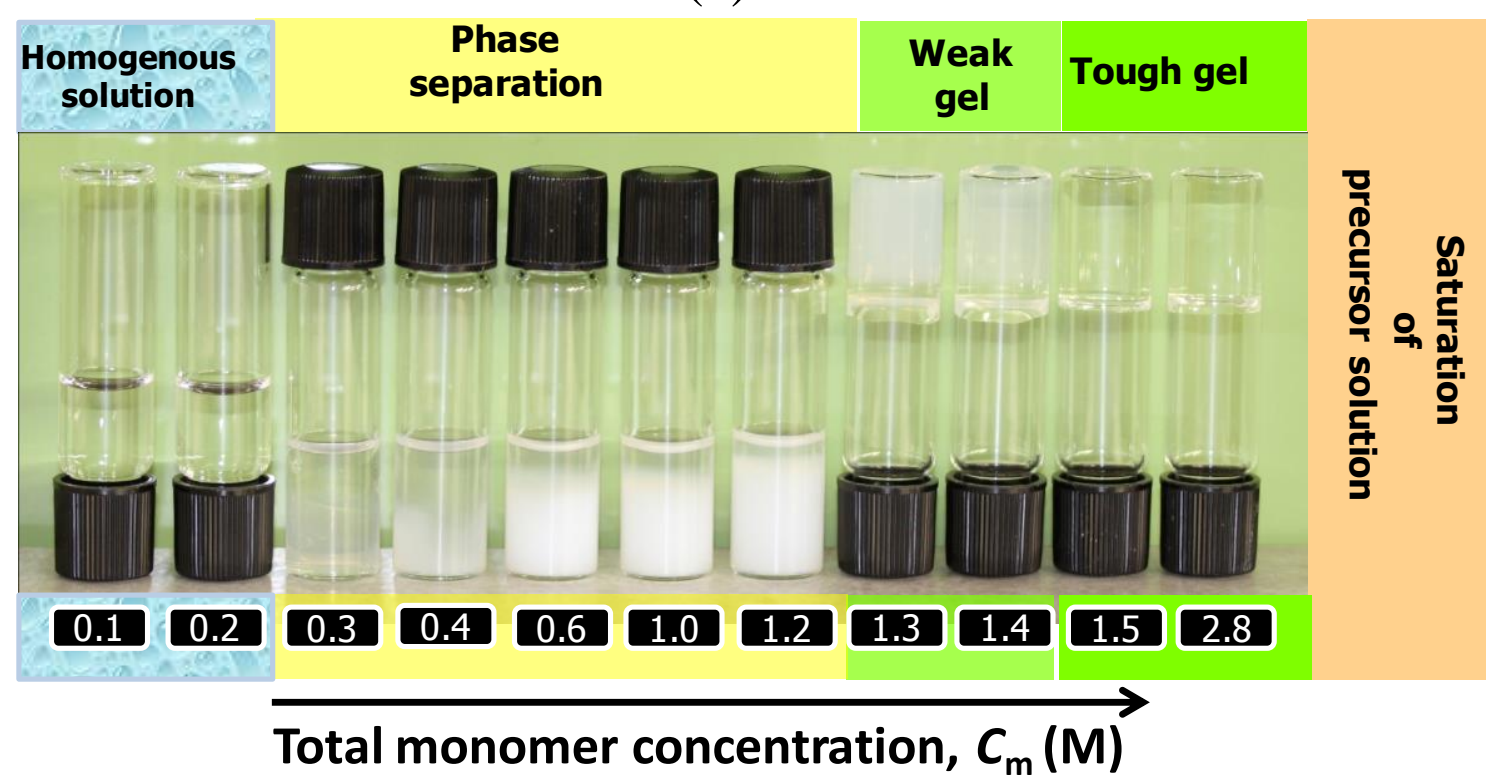

(b)

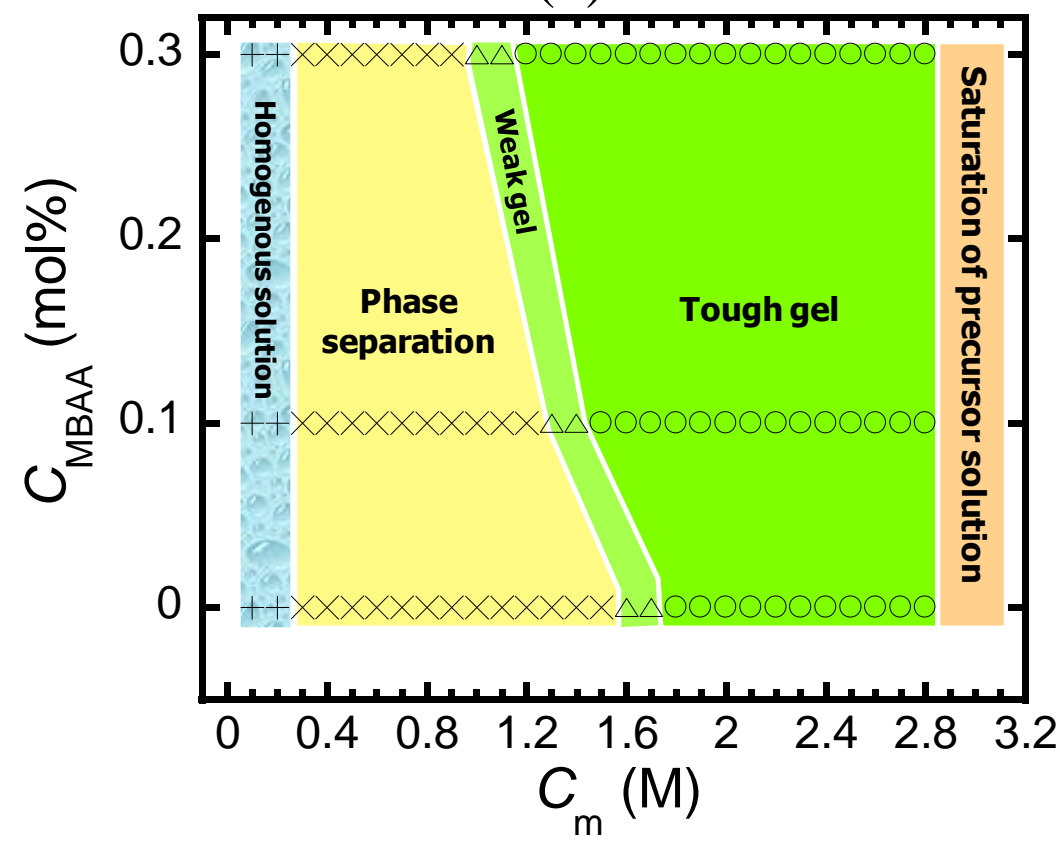

Fig. 2. (a) Photographs of as-prepared samples synthesized at various monomer concentrations $\left(C_{\mathrm{m}}\right)$ while keeping constant of anionic charge fraction $f=0.52\left(f_{\text {true }}=0.5\right)$ and chemical cross-linker density $C_{\mathrm{MBAA}}=0.1 \mathrm{~mol} \%$. (b) $C_{\mathrm{m}}-C_{\mathrm{MBAA}}$ diagram for $f=0.52$. 
Tough physical gels can be formed above a critical $C_{\mathrm{m}, \mathrm{c}}=1.8 \mathrm{M}$ even without chemical cross-linker. The tensile stress-strain curves of the polyampholyte physical gels synthesized at different $C_{\mathrm{m}}$ with no chemical cross-linker $\left(C_{\mathrm{MBAA}}=0\right)$ are shown in Fig. 3.

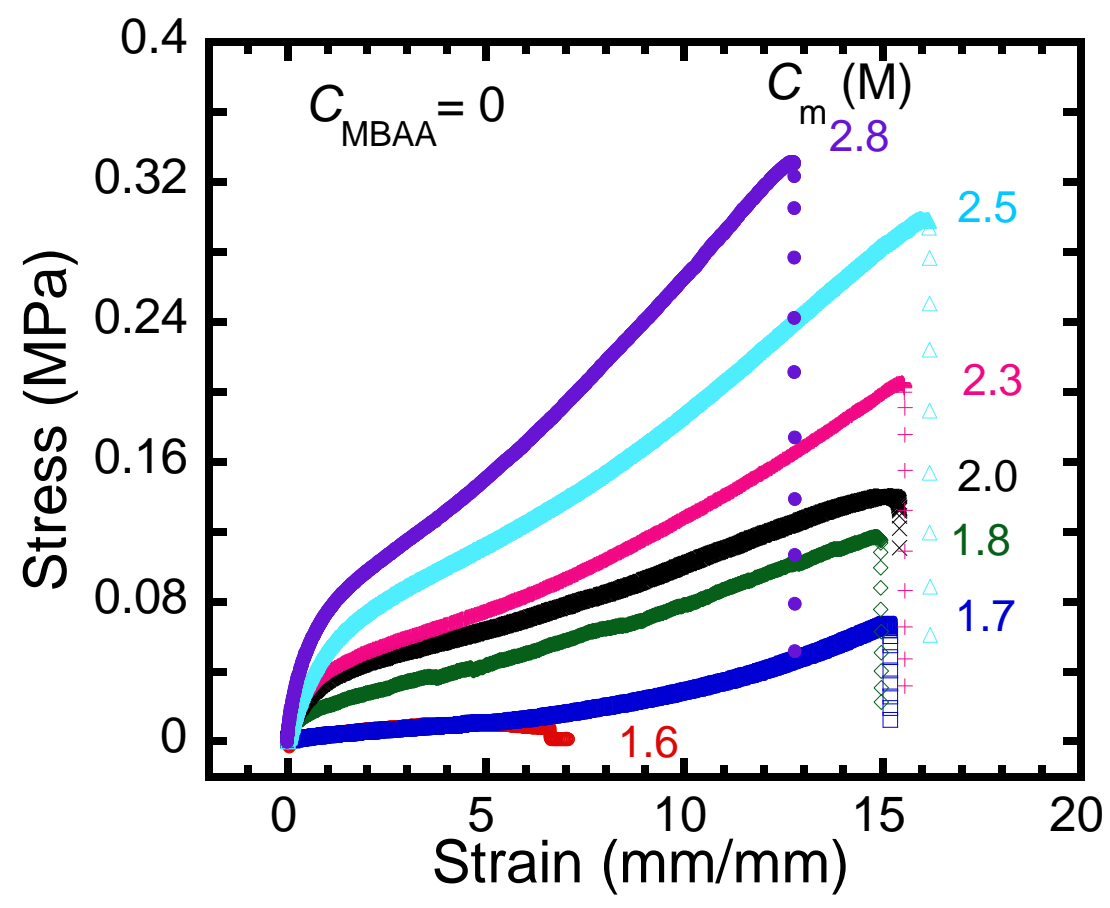

Fig. 3. Tensile stress-strain behaviors of the physical polyampholyte hydrogels $\left(C_{\mathrm{MBAA}}=0\right)$ synthesized at various $C_{\mathrm{m}}, \mathrm{P}(\mathrm{NaSS}-\mathrm{co}-\mathrm{DMAEA}-\mathrm{Q}) 0.52-C_{\mathrm{m}}-0$. 
Above the critical $C_{\mathrm{m}, \mathrm{c}}(=1.8 \mathrm{M})$, all the physical gels showed a very large fracture strain $\left(\varepsilon_{\mathrm{b}}>13-16\right)$, while the fracture stress increased with the increase in $C_{\mathrm{m}}$, reaching a value as high as $0.34 \mathrm{MPa}$ at $C_{\mathrm{m}}=2.8 \mathrm{M}$. Furthermore, the samples of low $C_{\mathrm{m}}(<1.9 \mathrm{M})$ did not show yielding point, indicating the weak ionic association, while the samples of high $C_{\mathrm{m}}(>1.9 \mathrm{M})$ exhibited clear yielding point. Apparently, the enhanced mechanical strength at high $C_{\mathrm{m}}$ is owing to the formation of inter-chain ionic associations, and the distinct yielding observed beyond a certain strain indicates the internal rupture of the relatively weak ion bonds. The final fracture of the sample should be attributed to the rupture of relatively strong ion bonds at large deformation.

Similar behaviors have been observed for $\mathrm{P}(\mathrm{NaSS}-\mathrm{co}-\mathrm{MTPC})$ physical gels that have a more hydrophobic cationic moiety, as reported in our the previous study. ${ }^{38}$ $\mathrm{P}(\mathrm{NaSS}-\mathrm{co}$-MTPC $)$ showed a much higher fracture stress $\left(\sigma_{\mathrm{b}}=1.8 \mathrm{MPa}\right)$ but much lower fracture strain $\left(\varepsilon_{\mathrm{b}}=7.4\right)$ than those of the $\mathrm{P}(\mathrm{NaSS}-\mathrm{co}$-DMAEA-Q) physical gels in this study.

We assume that the entanglement between the polymer chains, which is enhanced at high $\mathrm{C}_{\mathrm{m}}$, prevents the phase separation and leads to the formation of inter-chain ion bonds. Both of the effects favor for tough physical gel formation. This is justified by the influence of chemical cross-linker density, $C_{\mathrm{MBAA}}$, as depicted in Fig. 4 and 5. 


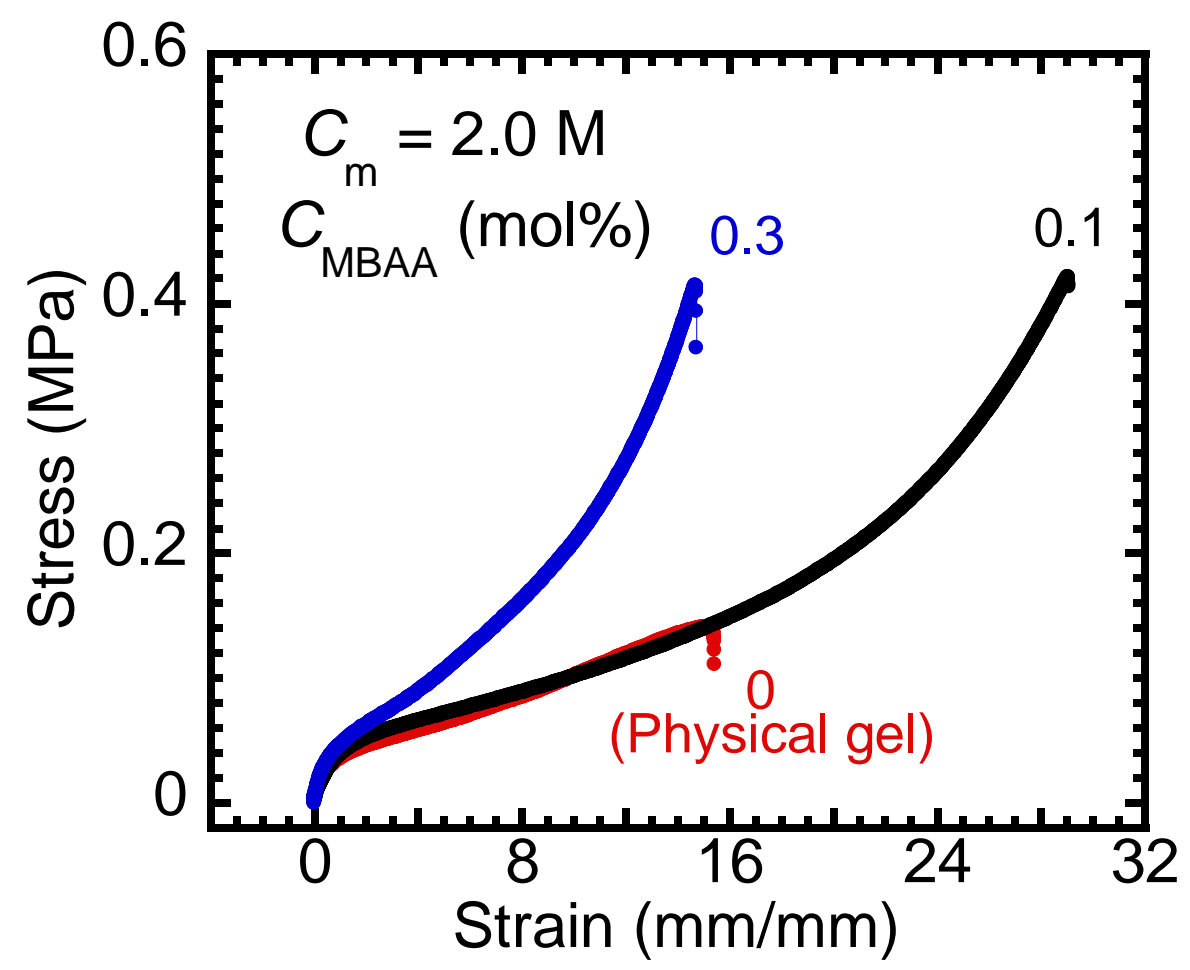

Fig. 4. Tensile stress-strain curves of polyampholyte hydrogels synthesized at various chemical cross-linker concentration $C_{\mathrm{MBAA}}$, P(NaSS-co-DMAEA-Q) 0.52-2.0- $C_{\text {MBAA}}$. 

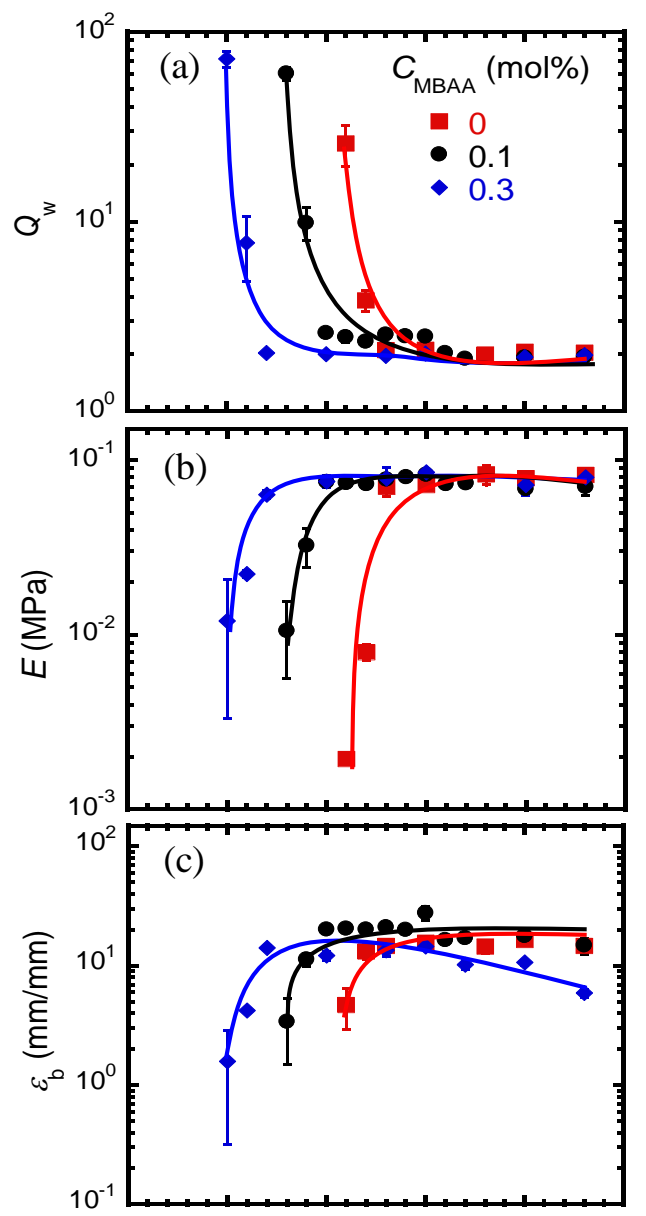

Fig. 5. Swelling ratio $Q_{\mathrm{w}}$ (inverse of polymer weight fraction) (a), modulus $E$ (b), fracture strain $\varepsilon_{\mathrm{b}}(\mathrm{c})$, fracture stress $\sigma_{\mathrm{b}}$ (d) and the work of extension at fracture $W_{\text {ext }}$ (toughness) (e) as a function of monomer concentration, $C_{\mathrm{m}}$, for samples with different $C_{\mathrm{MBAA}}$. Anionic charge fraction $f=0.52\left(f_{\text {true }}=0.5\right)$. Error bars are standard deviations from at least 5 samples.

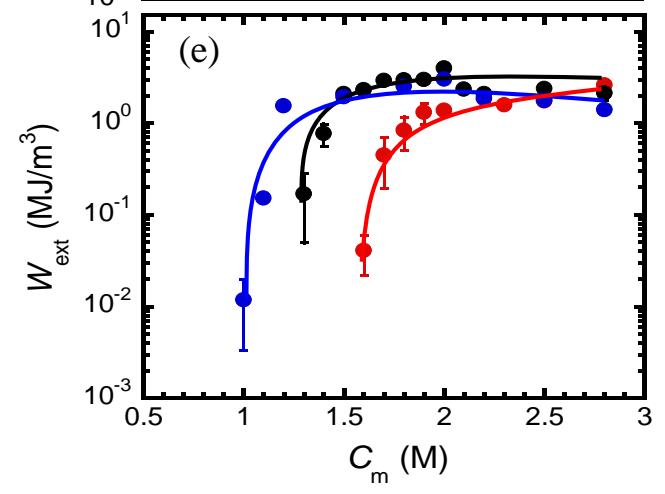


As shown in Fig. 5 (a), the weight swelling ratio $Q_{\text {w }}$ rapidly decreased with the increase in the monomer concentration $C_{\mathrm{m}}$ and reached the constant values above the $C_{\mathrm{m}, \mathrm{c}}$ even without adding the chemical cross-linker. In accompany with the change in $Q_{\mathrm{w}}$, the initial modulus $E$ of the gel remarkably increased with the increase in $C_{\mathrm{m}}$ and saturated to a constant value above the $C_{\mathrm{m}, \mathrm{c}}$ (Fig.5 (b)). These behaviors of $Q_{\mathrm{w}}$ and $E$ indicate that formation of stable inter-chain ion complex occurs above the critical $C_{\mathrm{m}, \mathrm{c}}$. The chemical cross-linker substantially promotes and stabilizes the ion complex formation. With the presence of the chemical cross-linker, the stable ion complex forms at a low critical $C_{\mathrm{m}, \mathrm{c}}(\mathbf{F i g . 5}(\mathbf{a}, \mathbf{b}))$. These results indicate that both the physical entanglement and the chemical cross-linking are able to stabilize the ion complex. Using $\mathrm{C}_{\mathrm{m}, \mathrm{c}}$ at various $\mathrm{C}_{\mathrm{MBAA}}$ determined from Figure 5, we obtained the boundary between the weak gel and tough gel in the phase diagram (Figure 2b).

For a constant $C_{\mathrm{m}}=2 \mathrm{M}$, addition of small amount of chemical cross-linker $\left(C_{\mathrm{MBAA}}=0.1 \mathrm{~mol} \%\right)$ hardly changed the stress-strain behavior below the fracture strain of the physical gel $\left(\varepsilon_{b}<16\right)$. However, it substantially enhanced the fracture stress and fracture strain, as shown in Fig. 4. This result indicate that the chemical cross-linker hardly influence the weak bond strength but can stabilize the strong bonds from rupture 
and enhance the strength and extensibility of the gel. Although both the swelling ratio $Q_{\mathrm{w}}$ and initial modulus $E$ are almost the same for the three samples of $C_{\mathrm{MBAA}}=0,0.1$, and $0.3 \mathrm{~mol} \%$, the larger amount of $C_{\mathrm{MBAA}}$ than $0.1 \mathrm{~mol} \%$ substantially limits the extensibility of the polymer chains, and lead to a small fracture strain (Fig. 5(c)). On the other hand, the fracture stress increases with the presence of the chemical cross-linker (Fig. 5(d)). The optimized structure of the gel is demonstrated by the work required to fracture the sample under tensile deformation, $W_{\text {ext }}$. The $W_{\text {ext }}$ of gels showed the maximum value at $C_{\mathrm{m}}=2.0 \mathrm{M}$ and $C_{\mathrm{MBAA}}=0.1 \mathrm{~mol} \% \quad(\mathbf{F i g} . \mathbf{5}(\mathbf{e}))$.

Based on the above results, the optimum formulation of the toughest gel is $f=0.52, C_{\mathrm{m}}=$ 2.0M and $C_{\mathrm{MBAA}}=0.1 \mathrm{~mol} \%$, coded as $\mathrm{P}(\mathrm{NaSS}-\mathrm{co}-\mathrm{DMEA}-\mathrm{Q}) 0.52-2.0-0.1($ Fig. 5(e)). This polyampholyte gel, containing $50 w t \%$ of water, shows a fracture stress of $\sigma_{\mathrm{b}} \sim 0.4$ MPa and fracture strain of $\varepsilon_{\mathrm{b}} \sim 30$ (Figure $\mathbf{5}(\mathbf{c}, \mathbf{d})$ ). The $W_{\text {ext }}$ of gel is calculated as 4 $\mathrm{MJ} / \mathrm{m}^{3}$ (Fig.5(e)), which is comparable to the super tough double network (DN) gel $\left(\right.$ several $\left.\mathrm{MJ} / \mathrm{m}^{3}\right){ }^{24}$

The $\mathrm{P}(\mathrm{NaSS}-$ co-DMEA-Q)0.52-2.0-0.1 is stable in $\mathrm{NaCl}$ solution below the concentration of $1 \mathrm{M}$. Above that concentration, the gel starts to swell and becomes weak due to dissociation of the ion complex. Detailed behaviors in high ionic strength medium will be given in a separate report. 


\section{Effect of ion bonds as reversible sacrificial bonds}

The random structures of polyampholyte hydrogels have been confirmed by ${ }^{1} \mathrm{H}-\mathrm{NMR}$ reaction kinetics study. The reactivity ratios were found to be $\mathrm{r}_{\mathrm{NaSS}}=2.41$ and $\mathrm{r}_{\text {DMAEA-Q }}=0.43$. These results indicate the random copolymerization of this polyampholyte with NaSS rich at the beginning of polymerization and long block sequences of DMAEA-Q at the end of polymerization due to their large difference of reactivity ratios, leading to the formation of different strength of ionic bonds. According to the sacrificial bond principle, the high strength and toughness of the polyampholyte gels is owing to the combination of the strong bonds and weak bonds. ${ }^{38}$ Under tensile deformation, the weak ion bonds, either intra-chains or inter-chains, are destroyed (sacrificial bond), and the polymer backbones in globule conformation are elongated extensively (hidden length).The former process involves yielding and irreversible energy dissipation, while the latter process permits the large deformation. After removal of stress, the stretched polymer chains return to the globule conformation, driven by elastic contraction (the rubber elasticity) and the reforming of ion complex between the same ion pairs of the intact sample. This process gives deformed sample the self-recovery to the intact state.

To justify this argument, we performed cyclic tensile test to observe the hysteresis of the 
deformation and its recovery, using the optimized sample, P(NaSS-co-DMAEA-Q) (0.52-2.0-0.1). As shown in Fig. 6a, a clear yielding is observed upon loading the sample to a predefined tensile strain, and a large hysteresis is observed upon unloading to zero stress. Furthermore, a residual strain was observed at the zero stress. The yielding reveals the structural change of the gel, i.e., dissociation of ion complex that dissipates energy. The residual strain and hysteresis reveal that the structural change is preserved within the unloading time. The residual strain decreased to zero after a certain waiting time.

To quantitatively characterize the time-dependent self-recovery, we performed multiple repeat of cyclic test at fixed maximum strain of 8 on the same sample with different time interval between two successive cycles (Fig. 6b). The first loading-unloading cycle of the virgin sample exhibits hysteresis. The immediate second loading-unloading cycle of the same sample with a time interval of 0 min shows partial recovery of the hysteresis loop. The hysteresis loops of subsequent cycles increase with the increase in time interval, and finally at an interval of $30 \mathrm{~min}$, the hysteresis is completely reproduced to that of virgin sample or first cycle, indicating the complete recovery of the gel strength and dimensions within $30 \mathrm{~min}$. The recovery ratio $\phi(\%)$, calculated from the hysteresis area ratio of the second to the first one, is shown in Fig. 6c for 
various recovery times.

(a)

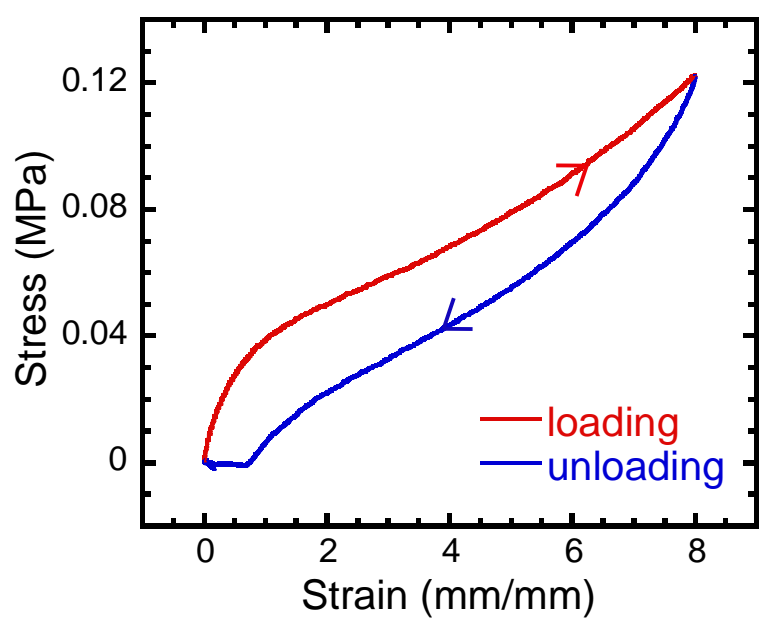

(b)

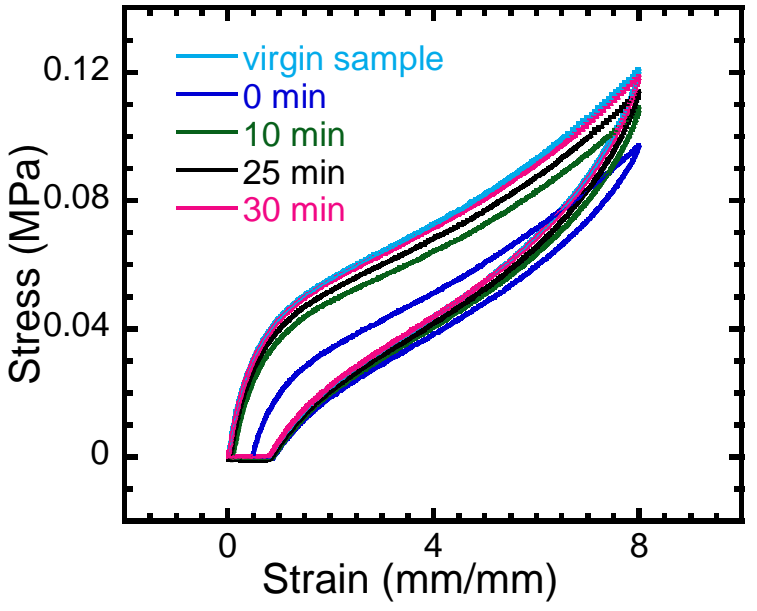

(c)

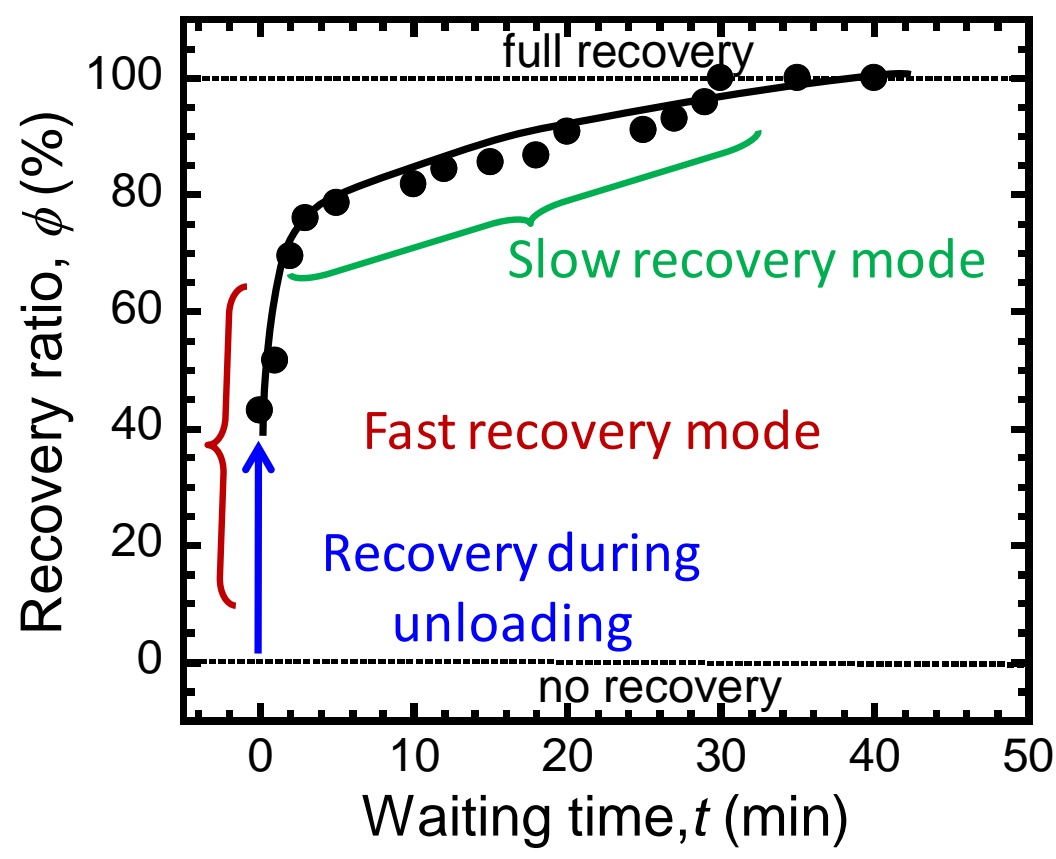

Fig. 6. Hysteresis and self-recovery of the polyampholyte hydrogel P(NaSS-co-DMAEA-Q) 0.5-2.0-0.1. (a) Tensile loading-unloading curves of the gel. (b) Tensile loading-unloading curves of the gel at various waiting times between first cycle and the second cycle. (c) The recovery ratio $\phi$ against the waiting time $t . \phi$ is defined as the area ratio of the hysteresis loop at different waiting times to the hysteresis loop of the virgin sample. 
At an interval of 0 min some extent of recovery can be noticed. Actually, this quick recovery occurred during the experimental unloading time ( $\sim 53 \mathrm{~s})$. Most of the hysteresis is recovered within $2 \mathrm{~min}$, and then the slow recovery process takes relatively longer time (30 min). The recovery is driven by the entropy gain of the stretched polymer chains to super-coiled state (rubber elasticity) while the ionic re-association during the recovery process should substantially slow-down the recovery kinetics (viscosity). The rubber elasticity is dominant in the fast recovery mode while the viscosity is dominant in the slow recovery mode. The $100 \%$ self-recovery of the gel indicates that no break of the main chain covalent bond occurs, and the energy dissipated during the cyclic test is due to the break of the fragile ion complex.

We also observed complete self-recovery of the physical gel P(NaSS-co-DMAEA-Q)0.5-2.0-0, i.e., residual strain decreased to zero after a certain waiting time ( $40 \mathrm{~min})$ when deformed up to a strain of 8 (data is not shown). This indicates that even without covalent cross-linking, no chain sliding occurs under the large deformation because of wide distributions of ion complex structures arising from the random copolymerization of the oppositely charged monomer. The strong ion complexes serve as permanent cross-linking points, and the weak ion complexes 
rupture as reversible sacrificial bonds that dissipate energy under load and recover upon unloading. The recovery of physical $\mathrm{P}(\mathrm{NaSS}-$ co-DMAEA-Q) gel is much faster than that of the more hydrophobic system $\mathrm{P}(\mathrm{NaSS}-\mathrm{co}-\mathrm{MPTC})$ that showed a much slow recovery time of $120 \mathrm{~min}^{38}$

The above results justify that the ionic bond in polyampholyte gels serves as a reversible sacrificial bond that breaks and reforms dynamically giving high toughness to this novel viscoelastic hydrogel. Although several high strength and toughness gels based on the physical association have recently been developed ${ }^{15,20,31}$, most of them only have partial self-recovery ability, probably due to some permanent damage of covalent bonds upon loading. Thus, the delicate balance between the physical bond of the rigid and brittle nature and the covalent bond of the soft and ductile main chain is crucial for the tough hydrogels.

\section{Conclusions}

Due to inter-chain ion bonds formation, the neutral polyampholyte $\mathrm{P}(\mathrm{NaSS}-\mathrm{co}$-DMAEA-Q) forms transparent and tough hydrogels above a critical monomer concentration $C_{\mathrm{m}, \mathrm{c}}$ even without chemical cross-linker $\left(C_{\mathrm{MBAA}}=0\right)$. Addition of chemical cross-linker promotes and stabilizes the ion bonds formation and therefore, shifts the critical $C_{\mathrm{m}, \mathrm{c}}$ to low values. Slight chemical cross-linking $\left(C_{\mathrm{MBAA}}=0.1 \mathrm{~mol} \%\right)$ 
substantially promote the toughness while a large amount of cross-linker $\left(C_{\mathrm{MBAA}}>\right.$ $0.1 \mathrm{~mol} \%$ ) limits the extensibility of the polymer chains and reduces the fracture strain. As a result, very tough and highly stretchable polyamphpolyte hydrogel, of supramolecular nature, is formed at an optimized composition of $C_{\mathrm{m}}=2.0 \mathrm{M}$ and $C_{\mathrm{MBAA}}=0.1 \mathrm{~mol} \%$. The polyampholyte gels containing dynamic and reversible ion bonds are viscoelastic, tough, and complete self-recoverable.

\section{Acknowledgements}

This research was financially supported by a Grant-in-Aid for Scientific Research (S) (No. 124225006) from Japan Society for the Promotion of Science (JSPS). 


\section{References}

(1) Y. Osada, H. Okuzaki, and H. Hori, Nature, 1992, 355, 242-244.

(2) R.Yoshida and T. Okano, Stimuli- responsive hydrogels and their application to functional materials, Biomedical applications of hydrogels handbook, Springer New York, 2010, 19-43.

(3) M. A. Meyers, P. Y. Chen, A. Y. M. Lin, and Y. Seki, Prog. Mater. Sci, 2008, 53, $1-206$.

(4) J. Kim, J. A. Hanna, M. Byun, C. D.Santangelo, and R. C. Hayward, Science, 2012, 335, 1201-1205.

(5) N. A. Peppas, J. Z. Hilt, A. Khademhosseini, and R. L. Peppas, Adv Mater, 2006, 18, $1345-1360$.

(6) Y. Osada and J. P. Gong, Adv Mater, 1998, 10, 827-837.

(7) A. P. Nowak, V. Breedveld, L. Pakstis, B. Ozbas, D.J. Pine, D. Pochan, and T. J. Deming, Nature, 2002, 417, 424-428.

(8) K.Y. Lee and D. J. Mooney, Chem. Rev., 2001, 101, 1869-1879.

(9) Y. Okumura and K. Ito, Adv Mater, 2001, 13, 485-487.

(10) K. Haraguchi and T. Takehisa, Adv Mater, 2002, 14, 1120-1124.

(11) J. P. Gong, Y. Katsuyama, T. Kurokawa, and Y. Osada, Adv Mater, 2003, 15, 
$1155-1158$.

(12) T. Huang, H. Xu, K. Jiao, L. Zhu, H. R. Brown, and H. Wang, Adv Mater, 2007, 19, $1622-1626$.

(13) T. Sakai, T. Matsunaga, Y. Yamamoto, C. Ito, R. Yoshida, S. Suzuki, N. Sasaki, M.

Shibayama, and U. I. Chung, Macromolecules, 2008, 41, 5379-5384.

(14) S. Naficy, H. R. Brown, J. M. Razal, G. M. Spinks, and P. G. Whitten, Aust. J. Chem., 2011, 64, 1007-1025.

(15) J. Y. Sun, X. Zhao, W. R. K. Illeperuma, O. Chaudhuri, K. H. Oh, D. J. Mooney, J. J. Vlassak, and Z. Suo, Nature, 2012, 489, 133-136.

(16) T. Nakajima, H. Sato, Y. Zhao, S. Kawahara, T. Kurokawa, K. Sugahara, and J. P. Gong, Adv Funct Mater, 2012, 22, 4426-4432.

(17) J. Hu, T. Kurokawa, T. Nakajima, T. L. Sun, T. Suekama, Z. L. Wu, S. M. Liang, and J. P. Gong, Macromolecules, 2012, 45, 9445-9451.

(18) J. Hu, K. Hiwatashi, T. Kurokawa, S. M. Liang, Z. L. Wu, and J. P. Gong, Macromolecules, 2011, 44, 7775-7781.

(19) J. Hu, T. Kurokawa, K. Hiwatashi, T. Nakajima, Z. L. Wu, S. M. Liang, and J. P. Gong, Macromolecules, 2012, 45, 5218-5228.

(20) M. A. Haque, T. Kurokawa, G. Kamita, and J. P. Gong, Macromolecules, 2011, 44, 
8916-8924.

(21) J. Saito, H. Furukawa, T. Kurokawa, R. Kuwabara, S. Kuroda, J. Hu, Y. Tanaka, J.

P. Gong, N. Kitamura, and K. Yasuda, Polym.Chem., 2011, 2, 575-580.

(22) D. Taylor, N. O'Mara, E. Ryan, M. Takaza, and C. Simms, J Mech Behav Biomed, 2012, 6, 139-147.

(23) Y. Tanaka, R. Kuwabara, Y.-H. Na, T. Kurokawa, J. P. Gong, and Y. Osada, J. Phys.

Chem. B, 2005, 109, 11559-11562.

(24) J. P. Gong, Soft Matter, 2010, 6, 2583-2590.

(25) Y.-H. Na, Y. Tanaka, Y. Kawauchi, H. Furukawa, T. Sumiyoshi, J. P. Gong, and Y. Osada, Macromolecules, 2006, 39, 4641-4645.

(26) R. E. Webber, C. Creton, H. R. Brown, and J. P. Gong, Macromolecules, 2007, 40, 2919-2927.

(27) Q. M. Yu, Y. Tanaka, H. Furukawa, T. Kurokawa, and J. P. Gong, Macromolecules, $2009, \mathbf{4 2}, 3852-3855$.

(28) T. Nakajima,T. Kurokawa, S. Ahmed, W. 1. Wu, and J. P. Gong, Soft Matter, 2013, 9, $1955-1966$.

(29) P. K. Hansma, P. J. Turner, and R. S. Ruoff, Nanotechnology, 2007, 18, 044026.

(30) G. E. Fantner, T. Hassenkam, J. H. Kindt, J. C. Weaver, H. Birkedal, L. Pechenik, J. 
A. Cutroni, G. A. G. Cidade, G. D. Stucky, D. E. Morse, and P. K. Hansma, Nat Mater, $2005,4,612-615$.

(31) K. J. Henderson, T. C. Zhou, K. J. Otim, and K. R. Shull, Macromolecules, 2010, 43, 6193-6201.

(32) S. E. Kudaibergenov, Adv. Polym Sci., 1999, 144, 115-197.

(33) P. G. Higgs and J. F. Joanny, J. Chem. Phys., 1991, 94, 1543-1554.

(34) G. Nisato, J. P. Munch, and S. J. Candau, Langmuir, 1999, 15, 4236-4244.

(35) Y. Takeoka, A. N. Berker, R. Du, T. Enoki, A. Grosberg, M. Kardar, T. Oya, K. Tanaka, G. Wang, X. Yu, and T. Tanaka, Phys. Rev. Lett., 1999, 82, 4863-4865.

(36) A. E. English, S. Mafe, J. A. Manzanares, X. Yu, A.Y. Grosberg, and T. Tanaka, J. Chem. Phys., 1996, 104, 8713-8720.

(37) K. S. Pafiti, Z. Philippou, E. Loizou, L. Porcar, and C. S. Patrickios, Macromolecules, 2011, 44, 5352-5362.

(38) T. L. Sun, T. Kurokawa, S. Kuroda, A.B. Ihsan, T. Akasaki, K. Sato, M.A. Haque, T. Nakajima, and J. P. Gong, Nature Materials, 2013, 2013, DOI: 10.1038/NMAT3713.

(39) T. Nakajima, H. Furukawa, Y. Tanaka, T. Kurokawa, Y. Osada, and J.P. Gong, Macromolecules, 2009, 42, 2184-2189.

(40) A. M. Bauer, A. P. Russell, and R. E. Shadwick, J. Exp. Biol., 1989, 145, 79-102.

(41) Y. C. Fung, Biomechanics: Mechanical properties of living tissues, 2nd ed.; Springer: New York, 1993. 
(42) H. Itagaki, T. Kurokawa, H. Furukawa, T. Nakajima, Y. Katsumoto, and J. P. Gong, Macromolecules, 2010, 43, 9495-9500. 
Table of Content (TOC)

A Phase Diagram of Neutral Polyampholyte - From Solution to Tough Hydrogel Abu Bin Ihsan ${ }^{1}$, Tao Lin Sun $^{2}$, Shinya Kuroda ${ }^{2}$, Md. Anamul Haque ${ }^{3}$, Takayuki Kurokawa $^{3}$, Tasuku Nakajima ${ }^{3}$, Jian Ping Gong**3



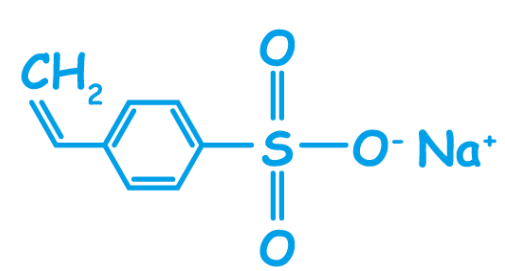

NaSS (-)<smiles>C=CC(=O)OCC[N+](C)(C)Cl</smiles>

DMAEA-Q (+)

Schematic illustration of the dynamic and reversible ionic bonds (weak bonds) formed by the random copolymerization of oppositely charged monomers at the charge balance point. 